\title{
Cross-intersecting families of partial permutations
}

\author{
Peter Borg \\ Department of Mathematics, University of Malta \\ Msida MSD 2080, Malta \\ p.borg.02@cantab.net
}

10th April 2010

\begin{abstract}
For positive integers $r$ and $n$ with $r \leq n$, let $\mathcal{P}_{n, r}$ be the family of all sets $\left\{\left(x_{1}, y_{1}\right), \ldots,\left(x_{r}, y_{r}\right)\right\}$ such that $x_{1}, \ldots, x_{r}$ are distinct elements of $[n]:=\{1, \ldots, n\}$ and $y_{1}, \ldots, y_{r}$ are also distinct elements of $[n] . \mathcal{P}_{n, n}$ describes permutations of $[n]$. For $r<n, \mathcal{P}_{n, r}$ describes $r$-partial permutations of $[n]$. Families $\mathcal{A}_{1}, \ldots, \mathcal{A}_{k}$ of sets are said to be cross-intersecting if, for any distinct $i$ and $j$ in $[k]$, any set in $\mathcal{A}_{i}$ intersects any set in $\mathcal{A}_{j}$. A sharp bound for the sum of sizes of cross-intersecting sub-families of $\mathcal{P}_{n, n}$ has recently been established by the author. We generalise this bound by showing that, if $\mathcal{A}_{1}, \ldots, \mathcal{A}_{k}$ are cross-intersecting sub-families of $\mathcal{P}_{n, r}$, then

$$
\sum_{i=1}^{k}\left|\mathcal{A}_{i}\right| \leq \begin{cases}\left(\begin{array}{l}
n \\
r
\end{array}\right) \frac{n !}{(n-r) !} & \text { if } k \leq \frac{n^{2}}{r} \\
k\left(\begin{array}{l}
n-1 \\
r-1
\end{array}\right) \frac{(n-1) !}{(n-r) !} & \text { if } k \geq \frac{n^{2}}{r}\end{cases}
$$

We also determine the structures for which the bound is attained when $r<n$. Our main tool is an extension of Katona's cyclic permutation method.
\end{abstract}

\section{Introduction}

For an integer $n$, the set $\{1,2 \ldots, n\}$ is denoted by $[n]$. The power set $\{A: A \subseteq X\}$ of a set $X$ is denoted by $2^{X}$, and the uniform sub-family $\{Y \subseteq X:|Y|=r\}$ is denoted by $\left(\begin{array}{c}X \\ r\end{array}\right)$.

If $\mathcal{F}$ is a family of sets and $x$ is an element of the union of all sets in $\mathcal{F}$, then we call the sub-family of $\mathcal{F}$ consisting of those sets that contain $x$ a star of $\mathcal{F}$ with centre $x$.

A family $\mathcal{A}$ is said to be intersecting if any two sets in $\mathcal{A}$ intersect. Note that a star of a family is trivially intersecting.

The classical Erdôs-Ko-Rado (EKR) Theorem [12] says that, if $r \leq n / 2$, then an intersecting sub-family $\mathcal{A}$ of $\left(\begin{array}{c}{[n]} \\ r\end{array}\right)$ has size at most $\left(\begin{array}{c}n-1 \\ r-1\end{array}\right)$, i.e. the size of a star of $\left(\begin{array}{c}{[n]} \\ r\end{array}\right)$; if $r<n / 2$, then $\mathcal{A}$ attains the bound if and only if $\mathcal{A}$ is a star of $\left(\begin{array}{c}{[n]} \\ r\end{array}\right)$ (see $\left.[12,15]\right)$. Two alternative proofs of the EKR Theorem that are particularly short and beautiful were obtained by 
Katona [16] and Daykin [7]. In his proof, Katona introduced an elegant averaging technique called the cycle method. Daykin's proof is based on a fundamental result known as the Kruskal-Katona Theorem [17, 18]. The EKR Theorem inspired a wealth of results and continues to do so; the survey papers $[9,13]$ are recommended.

For integers $r$ and $n$ with $r \leq n$, let

$$
\begin{array}{r}
\mathcal{P}_{n, r}:=\left\{\left\{\left(x_{1}, y_{1}\right), \ldots,\left(x_{r}, y_{r}\right)\right\}: x_{1}, \ldots, x_{r} \text { are distinct elements of }[n],\right. \\
\left.y_{1}, \ldots, y_{r} \text { are distinct elements of }[n]\right\} .
\end{array}
$$

$\mathcal{P}_{n, n}$ describes permutations of the set $[n]$ because a permutation $y_{1} y_{2} \ldots y_{n}$ of $[n]$ corresponds uniquely to the set $\left\{\left(1, y_{1}\right),\left(2, y_{2}\right), \ldots,\left(n, y_{n}\right)\right\}$ in $\mathcal{P}_{n, n}$. An r-partial permutation of a set $[n]$ is an ordered pair $(A, f)$ where $A \in\left(\begin{array}{c}{[n]} \\ r\end{array}\right)$ and $f: A \rightarrow[n]$ is an injection (see [19]). So $\mathcal{P}_{n, r}$ describes $r$-partial permutations because an $r$-partial permutation $\left(\left\{a_{1}, \ldots, a_{r}\right\}, f\right)$ corresponds uniquely to the set $\left\{\left(a_{1}, f\left(a_{1}\right)\right), \ldots,\left(a_{r}, f\left(a_{r}\right)\right)\right\}$ in $\mathcal{P}_{n, r}$; also, if two sets $\left\{\left(a_{1}, f\left(a_{1}\right)\right), \ldots,\left(a_{r}, f\left(a_{r}\right)\right)\right\}$ and $\left\{\left(b_{1}, g\left(b_{1}\right)\right), \ldots,\left(b_{r}, g\left(b_{r}\right)\right)\right\}$ in $\mathcal{P}_{n, r}$ intersect, then $a_{i}=b_{j}$ and $f\left(a_{i}\right)=g\left(b_{j}\right)$ for some $i, j \in[r]$, which is exactly what we mean when we say that the $r$-partial permutations $\left(\left\{a_{1}, \ldots, a_{r}\right\}, f\right)$ and $\left(\left\{b_{1}, \ldots, b_{r}\right\}, g\right)$ intersect (see $\left.[6,19]\right)$.

Deza and Frankl [8] proved an analogue of the EKR Theorem for permutations. Cameron and $\mathrm{Ku} \mathrm{[6]} \mathrm{and} \mathrm{Larose} \mathrm{and} \mathrm{Malvenuto} \mathrm{[20]} \mathrm{independently} \mathrm{showed} \mathrm{that} \mathrm{the} \mathrm{largest} \mathrm{intersect-}$ ing sub-families of $\mathcal{P}_{n, n}$ are the stars of $\mathcal{P}_{n, n}$.

Theorem $1.1([8,6,20])$ If $\mathcal{A}$ is an intersecting sub-family of $\mathcal{P}_{n, n}$, then $|\mathcal{A}| \leq(n-1)$ !, and equality holds if and only if $\mathcal{A}$ is a star of $\mathcal{P}_{n, n}$.

$\mathrm{Ku}$ and Leader [19] solved the EKR problem for $r$-partial permutations of $[n]$ using Katona's cycle method. They also proved that, for $8 \leq r \leq n-3$, the largest intersecting sub-families of $\mathcal{P}_{n, r}$ are the stars, and they conjectured that only the stars are extremal for the few remaining values of $r$ too. Li and Wang [21] proved this conjecture, taking the same cycle method approach of Ku and Leader.

Theorem $1.2([\mathbf{1 9}, \mathbf{2 1}])$ If $1 \leq r<n$ and $\mathcal{A}$ is an intersecting sub-family of $\mathcal{P}_{n, r}$, then $|\mathcal{A}| \leq\left(\begin{array}{l}n-1 \\ r-1\end{array}\right) \frac{(n-1) !}{(n-r) !}$, and equality holds if and only if $\mathcal{A}$ is a star of $\mathcal{P}_{n, r}$.

We now shift our attention to cross-intersecting families. Families $\mathcal{A}_{1}, \ldots, \mathcal{A}_{k}$ are said to be cross-intersecting if, for any $i, j \in[k]$ such that $i \neq j$, any set in $\mathcal{A}_{i}$ intersects any set in $\mathcal{A}_{j}$. The study of cross-intersecting families is related to the study of intersecting families, and results for cross-intersecting families are generally stronger than results for intersecting families; this will become clear from the rest of the paper.

Hilton [14] established the following best possible cross-intersection result using the Kruskal-Katona Theorem.

Theorem $1.3\left([\mathbf{1 4 ]})\right.$ Let $r \leq n / 2$ and $k \geq 2$. Let $\mathcal{A}_{1}, \ldots, \mathcal{A}_{k}$ be cross-intersecting subfamilies of $\left(\begin{array}{c}{[n]} \\ r\end{array}\right)$. Then

$$
\sum_{i=1}^{k}\left|\mathcal{A}_{i}\right| \leq \begin{cases}\left(\begin{array}{l}
n \\
r
\end{array}\right) & \text { if } k \leq \frac{n}{r} \\
k\left(\begin{array}{l}
n-1 \\
r-1
\end{array}\right) & \text { if } k \geq \frac{n}{r}\end{cases}
$$


Unless $k=2=n / r$, the bound is attained if and only if one of the following holds:

(i) $k<n / r, \mathcal{A}_{i}=\left(\begin{array}{c}{[n]} \\ r\end{array}\right)$ for some $i \in[k]$, and $\mathcal{A}_{j}=\emptyset$ for all $j \in[k] \backslash\{i\}$;

(ii) $k>n / r$ and $\left|\mathcal{A}_{1}\right|=\ldots=\left|\mathcal{A}_{k}\right|=\left(\begin{array}{c}n-1 \\ r-1\end{array}\right)$;

(iii) $k=n / r$ and $\mathcal{A}_{1}, \ldots, \mathcal{A}_{k}$ are as in (i) or (ii).

The EKR Theorem follows from this result: take $\mathcal{A}$ to be any intersecting sub-family of $\left(\begin{array}{c}{[n]} \\ r\end{array}\right)$, and take $\mathcal{A}_{1}=\ldots=\mathcal{A}_{k}=\mathcal{A}$ and $k>n / r$ in the above result.

For any family $\mathcal{A}$, we define $\mathcal{A}^{*}$ to be the sub-family of $\mathcal{A}$ consisting of those sets in $\mathcal{A}$ that intersect each set in $\mathcal{A}$, and we set $\mathcal{A}^{\prime}:=\mathcal{A} \backslash \mathcal{A}^{*}$. So $\mathcal{A}^{\prime}$ consists of those sets in $\mathcal{A}$ that do not intersect all sets in $\mathcal{A}$.

In [2], the following extension of the EKR Theorem is proved and shown to immediately yield Theorem 1.3 (it is also shown that in case (ii) of Theorem 1.3, we actually have that $\mathcal{A}_{1}=\ldots=\mathcal{A}_{k}$ and $\mathcal{A}_{1}$ is a star of $\left.\left(\begin{array}{c}{[n]} \\ r\end{array}\right)\right)$.

Theorem 1.4 ([2]) Let $r \leq n / 2$, and let $\mathcal{A} \subseteq\left(\begin{array}{c}{[n]} \\ r\end{array}\right)$. Then

$$
\left|\mathcal{A}^{*}\right|+\frac{r}{n}\left|\mathcal{A}^{\prime}\right| \leq\left(\begin{array}{c}
n-1 \\
r-1
\end{array}\right)
$$

and if $n>2 r$ then equality holds if and only if either $\mathcal{A}^{\prime}=\left(\begin{array}{c}{[n]} \\ r\end{array}\right)$ and $\mathcal{A}^{*}=\emptyset$ or $\mathcal{A}^{\prime}=\emptyset$ and $\mathcal{A}^{*}$ is a star of $\left(\begin{array}{c}{[n]} \\ r\end{array}\right)$.

The proof was obtained by extending Daykin's proof of the EKR Theorem.

As explained below, in this paper we provide an analogue of Theorem 1.4 for partial permutations and use it to obtain an analogue of Theorem 1.3 also for partial permutations.

A sharp bound for the sum of sizes of cross-intersecting families of permutations emerges from [4, Theorem 2.4], and the solution to the problem of determining precisely the cases (conjectured in [4]) in which the bound is attained is given in [3] as part of a more general result for permutations of subsets of $[n]$.

Theorem $1.5([3,4])$ If $\mathcal{A}_{1}, \ldots, \mathcal{A}_{k}$ are cross-intersecting sub-families of $\mathcal{P}_{n, n}$, then

$$
\sum_{i=1}^{k}\left|\mathcal{A}_{i}\right| \leq \begin{cases}n ! & \text { if } k \leq n \\ k(n-1) ! & \text { if } k \geq n\end{cases}
$$

Unless $2 \leq k \leq 3=n$, the bound is attained if and only if one of the following holds:

(i) $k<n, \mathcal{A}_{i}=\mathcal{P}_{n, n}$ for some $i \in[k]$, and $\mathcal{A}_{j}=\emptyset$ for all $j \in[k] \backslash\{i\}$;

(ii) $k>n, \mathcal{A}_{1}=\ldots=\mathcal{A}_{k}$ and $\mathcal{A}_{1}$ is a star of $\mathcal{P}_{n, n}$;

(iii) $k=n$ and $\mathcal{A}_{1}, \ldots, \mathcal{A}_{k}$ are as in (i) or (ii).

In the case $2 \leq k \leq 3=n$ there are other optimal configurations (see [3]).

We mention that other authors have considered the maximum product problem (see [10], [11]); in [10] it is proved that the product of sizes of any $k \geq 2$ cross-intersecting 
sub-families of $\mathcal{P}_{n, n}$ is a maximum if they are all the same star of $\mathcal{P}_{n, n}$. In this paper we are interested in the maximum sum problem.

Our aim is to extend Theorem 1.5 to a result for cross-intersecting sub-families of $\mathcal{P}_{n, r}$ and to also determine the extremal structures. The case $r<n$ requires methods that are different from the ones in [3,4] for the special case $r=n$. Using the method in [5], which provides an extension of Katona's cycle method [16], and tools forged by $\mathrm{Ku}$ and Leader [19] (inspired by the idea of good cyclic orderings in [1]), we first prove the following analogue of Theorem 1.4.

Theorem 1.6 Let $r<n$, and let $\mathcal{A} \subseteq \mathcal{P}_{n, r}$. Then

$$
\left|\mathcal{A}^{*}\right|+\frac{r}{n^{2}}\left|\mathcal{A}^{\prime}\right| \leq\left(\begin{array}{c}
n-1 \\
r-1
\end{array}\right) \frac{(n-1) !}{(n-r) !},
$$

and equality holds if and only if one of the following holds:

(i) $\mathcal{A}^{\prime}=\mathcal{P}_{n, r}$ and $\mathcal{A}^{*}=\emptyset$;

(ii) $\mathcal{A}^{\prime}=\emptyset$ and $\mathcal{A}^{*}$ is a star of $\mathcal{P}_{n, r}$.

We then apply an argument found in $[2,3]$ to show that the above result enables us to extend Theorem 1.5 to the following analogue of Theorem 1.3 for permutations and partial permutations.

Theorem 1.7 Let $r \leq n, k \geq 2$. Let $\mathcal{A}_{1}, \ldots, \mathcal{A}_{k}$ be cross-intersecting sub-families of $\mathcal{P}_{n, r}$. Then

$$
\sum_{i=1}^{k}\left|\mathcal{A}_{i}\right| \leq \begin{cases}\left(\begin{array}{l}
n \\
r
\end{array}\right) \frac{n !}{(n-r) !} & \text { if } k \leq \frac{n^{2}}{r} \\
k\left(\begin{array}{l}
n-1 \\
r-1
\end{array}\right) \frac{(n-1) !}{(n-r) !} & \text { if } k \geq \frac{n^{2}}{r}\end{cases}
$$

Unless $2 \leq k \leq 3=r=n$, the bound is attained if and only if one of the following holds:

(i) $k<\frac{n^{2}}{r}, \mathcal{A}_{i}=\mathcal{P}_{n, r}$ for some $i \in[k]$, and $\mathcal{A}_{j}=\emptyset$ for all $j \in[k] \backslash\{i\}$;

(ii) $k>\frac{n^{2}}{r}, \mathcal{A}_{1}=\ldots=\mathcal{A}_{k}$ and $\mathcal{A}_{1}$ is a star of $\mathcal{P}_{n, r}$;

(iii) $k=\frac{n^{2}}{r}$ and $\mathcal{A}_{1}, \ldots, \mathcal{A}_{k}$ are as in (i) or (ii).

This generalises Theorems 1.1 and 1.2: take $k>n^{2} / r$ and $\mathcal{A}_{1}=\ldots=\mathcal{A}_{k}=\mathcal{A}$, where $\mathcal{A}$ is an arbitary intersecting sub-family of $\mathcal{P}_{n, r}$, in the above result.

\section{Proofs}

In various parts of this paper we will use ' $\bmod ^{*}$ ' to represent the usual modulo operation with the exception that, for any two integers $a$ and $b, b a \bmod ^{*} a$ is $a$ instead of 0 . For convenience we shall abbreviate $(b+c) \bmod ^{*} a$ to $b+c \bmod ^{*} a$, that is, $b+c \bmod ^{*} a$ is the result of applying the $\bmod ^{*} c$ operation to $(a+b)$.

Let $X$ be an $m$-set. A cyclic ordering of $X$ is a bijection $\sigma: X \rightarrow[m]$. An $r$ interval of $\sigma$ is a sequence $\sigma^{-1}(i), \sigma^{-1}\left(i+1 \bmod ^{*} m\right), \ldots, \sigma^{-1}\left(i+r-1 \bmod ^{*} m\right)$ for some $i \in[m]$, that is, the sequence of those $r$ elements $x_{1}, x_{2}, \ldots, x_{r}$ of $X$ that are mapped to 
$i, i+1 \bmod ^{*} m, \ldots, i+r-1 \bmod ^{*} m$ (respectively) by $\sigma$. We say that an $r$-subset $A$ of $X$ meets $\sigma$ if $A$ consists of the points of some $r$-interval of $\sigma$.

The lemma below, which is inspired by Theorem 1.4, will be our main tool. It extends Katona's cycle method by giving a result for any family, whether intersecting or not. The lemma is established in [5], but we shall give the proof for completeness since it is short.

Lemma 2.1 ([5]) Let $m \geq 2 r$, and let $X$ be a set of size $m$. Let $\sigma$ be a cyclic ordering of $X$. Let $\mathcal{C}$ be the family of those r-subsets of $X$ that meet $\sigma$. For any $\mathcal{B} \subseteq \mathcal{C}$ we have

$$
\left|\mathcal{B}^{*}\right|+\frac{r}{m}\left|\mathcal{B}^{\prime}\right| \leq r
$$

and if $m>2 r$ then equality holds if and only if either $\mathcal{B}^{\prime}=\mathcal{C}$ and $\mathcal{B}^{*}=\emptyset$ or $\mathcal{B}^{\prime}=\emptyset$ and $\left|\mathcal{B}^{*}\right|=r$.

Proof. Clearly there are $m r$-subsets of $X$ that meet $\sigma$, i.e. $|\mathcal{C}|=m$. So the result is straightforward if $\mathcal{B}^{*}=\emptyset$. Suppose $\mathcal{B}^{*} \neq \emptyset$. Let $B^{*} \in \mathcal{B}^{*}$, and let $x_{1}, \ldots, x_{r}$ be the $r$-interval of $\sigma$ such that $B^{*}=\left\{x_{1}, \ldots, x_{r}\right\}$. For $i \in[r]$, let $C_{i} \in \mathcal{C}$ be the set of points in the $r$-interval of $\sigma$ starting with $x_{i}$, and let $C_{i}^{\prime} \in \mathcal{C}$ be the set of points in the $r$-interval of $\sigma$ ending with $x_{i}$. Let $\mathcal{D}:=\left\{C_{1}, \ldots, C_{r}\right\} \cup\left\{C_{1}^{\prime}, \ldots, C_{r}^{\prime}\right\}$. Note that $B^{*}=C_{1}=C_{r}^{\prime}$ and hence $\mathcal{D}=\left\{B^{*}\right\} \cup\left\{C_{2}, \ldots, C_{r}\right\} \cup\left\{C_{1}^{\prime}, \ldots, C_{r-1}^{\prime}\right\}$. By the definitions of $\mathcal{B}^{*}$ and $\mathcal{B}^{\prime}$, we have $\mathcal{B}^{*} \cup \mathcal{B}^{\prime} \subseteq \mathcal{D}$ (because $\mathcal{D}$ consists of all the sets in $\mathcal{C}$ that intersect $B^{*}$, and each set in $\mathcal{B}=\mathcal{B}^{*} \cup \mathcal{B}^{\prime}$ intersects $\left.B^{*}\right)$ and, since $r \leq m / 2, C_{j-1}^{\prime} \notin \mathcal{B}^{*} \cup \mathcal{B}^{\prime}$ for any $j \in\{2, \ldots, r\}$ such that $C_{j} \in \mathcal{B}^{*}$. It follows that there are at least $\left|\mathcal{B}^{*}\right|-1$ sets in $\mathcal{D}$ that are not in $\mathcal{B}^{*} \cup \mathcal{B}^{\prime}$, and hence $\left|\mathcal{B}^{\prime}\right| \leq|\mathcal{D}|-\left|\mathcal{B}^{*}\right|-\left(\left|\mathcal{B}^{*}\right|-1\right)=2 r-2\left|\mathcal{B}^{*}\right|$. So

$$
\left|\mathcal{B}^{*}\right|+\frac{r}{m}\left|\mathcal{B}^{\prime}\right| \leq\left|\mathcal{B}^{*}\right|+\frac{1}{2}\left|\mathcal{B}^{\prime}\right| \leq\left|\mathcal{B}^{*}\right|+\frac{1}{2}\left(2 r-2\left|\mathcal{B}^{*}\right|\right)=r
$$

and it is immediate from this expression that if $\frac{r}{m}<\frac{1}{2}$ then equality holds throughout if and only if $\left|\mathcal{B}^{*}\right|=r$ and $\mathcal{B}^{\prime}=\emptyset$. Hence the result.

Katona [16] proved the above result for intersecting sub-families of $\mathcal{C}$. Our result applies to any sub-family.

Each set in $\mathcal{P}_{n, r}$ is a subset of the Cartesian product $[n] \times[n]$. We say that a cyclic ordering $\sigma$ of $[n] \times[n]$ is $r$-good if any $r$-interval of $\sigma$ forms a set in $\mathcal{P}_{n, r}$; in simple terms, $\sigma$ is $r$-good if any $r$ elements $\left(x_{1}, y_{1}\right), \ldots,\left(x_{r}, y_{r}\right)$ of $[n] \times[n]$ that are numbered consecutively (in the cyclic sense) by $\sigma$ are such that $x_{1}, \ldots, x_{r}$ are distinct and $y_{1}, \ldots, y_{r}$ are distinct. Note that, if a cyclic ordering is $r$-good, then it is $s$-good for all $s \in[r]$.

It is easy to see that no cyclic ordering of $[n] \times[n]$ is $n$-good. However, Ku and Leader [19] observed that the cyclic ordering $\tau:[n] \times[n] \rightarrow\left[n^{2}\right]$ defined by $\tau(x, y)=n\left(y-x \bmod ^{*} n\right)+x$ is $r$-good for all $r \in[n-1]$. In the proof of Theorem 1.6, we shall instead use the cyclic ordering $\gamma:[n] \times[n] \rightarrow\left[n^{2}\right]$ defined by

$$
\gamma(x, y)=n\left(x-y \bmod ^{*} n\right)+y
$$


$\gamma$ is also $r$-good for all $r \in[n-1]$. This can be immediately seen from the following example with $n=5$, where each element $(x, y)$ of $[n] \times[n]$ is given the label $\gamma(x, y)$ shown in bold superscript.

$\begin{array}{lllll}(1,5)^{\mathbf{1 0}} & (2,5)^{\mathbf{1 5}} & (3,5)^{\mathbf{2 0}} & (4,5)^{\mathbf{2 5}} & (5,5)^{\mathbf{5}} \\ (1,4)^{\mathbf{1 4}} & (2,4)^{\mathbf{1 9}} & (3,4)^{\mathbf{2 4}} & (4,4)^{\mathbf{4}} & (5,4)^{\mathbf{9}} \\ (1,3)^{\mathbf{1 8}} & (2,3)^{\mathbf{2 3}} & (3,3)^{\mathbf{3}} & (4,3)^{\mathbf{8}} & (5,3)^{\mathbf{1 3}} \\ (1,2)^{\mathbf{2 2}} & (2,2)^{\mathbf{2}} & (3,2)^{\mathbf{7}} & (4,2)^{\mathbf{1 2}} & (5,2)^{\mathbf{1 7}} \\ (1,1)^{\mathbf{1}} & (2,1)^{\mathbf{6}} & (3,1)^{\mathbf{1 1}} & (4,1)^{\mathbf{1 6}} & (5,1)^{\mathbf{2 1}}\end{array}$

It may be very helpful to keep the above illustration in mind when reading the proof of the theorem, particularly the part dealing with the extremal cases.

Let $S_{n}$ be the set of bijections from $[n]$ to $[n]$. For $\pi, \rho \in S_{k}$ and a cyclic ordering $\sigma$ of $[n] \times[n]$, let $\sigma_{\pi, \rho}:[n] \times[n] \rightarrow\left[n^{2}\right]$ be defined by $\sigma_{\pi, \rho}(\pi(i), \rho(j))=\sigma(i, j)$ for any $i, j \in[n]$. Note that $\sigma_{\pi, \rho}$ is a cyclic ordering of $[n] \times[n]$. Moreover, the following holds.

Lemma 2.2 Let $r \in[n-1]$, and let $\sigma$ be an $r$-good cyclic ordering of $[n] \times[n]$. Then, for any $\pi, \rho \in S_{n}, \sigma_{\pi, \rho}$ is an r-good cyclic ordering of $[n] \times[n]$.

Proof. Suppose not. Then there exists an $r$-interval $\left(x_{1}, y_{1}\right), \ldots,\left(x_{r}, y_{r}\right)$ of $\sigma_{\pi, \rho}$ such that either $x_{1}, \ldots, x_{r}$ are not all distinct or $y_{1}, \ldots, y_{r}$ are not all distinct. So there exist two distinct elements $\left(a_{1}, b_{1}\right)$ and $\left(a_{2}, b_{2}\right)$ of $\left\{\left(x_{1}, y_{1}\right), \ldots,\left(x_{r}, y_{r}\right)\right\}$ and an integer $p \in[r-1]$ such that $\sigma_{\pi, \rho}\left(a_{2}, b_{2}\right)=\sigma_{\pi, \rho}\left(a_{1}, b_{1}\right)+p \bmod ^{*} n^{2}$ and either $a_{1}=a_{2}\left(\right.$ and $\left.b_{1} \neq b_{2}\right)$ or $b_{1}=b_{2}$ (and $\left.a_{1} \neq a_{2}\right)$. Suppose $a_{1}=a_{2}$. Setting $i:=\pi^{-1}\left(a_{1}\right), j_{1}:=\rho^{-1}\left(b_{1}\right), j_{2}:=\rho^{-1}\left(b_{2}\right)$, we get $\sigma\left(i, j_{2}\right)=\sigma\left(i, j_{1}\right)+p \bmod ^{*} n^{2}$, which contradicts the fact that $\sigma$ is an $r$-good cyclic ordering of $[n] \times[n]$. Similarly, we cannot have $b_{1}=b_{2}$.

Proof of Theorem 1.6. We assume $r \geq 2$ and $n \geq 3$ as otherwise the result is trivial. Let $X:=[n] \times[n]$ and $\mathcal{P}:=\mathcal{P}_{n, r}$. For a cyclic ordering $\sigma$ of $X$, a family $\mathcal{F} \subseteq \mathcal{P}$ and a set $P \in \mathcal{P}$, let $\mathcal{F}_{\sigma}=\{F \in \mathcal{F}: F$ meets $\sigma\}$ and

$$
\Phi(\sigma, P)= \begin{cases}1 & \text { if } P \text { meets } \sigma \\ 0 & \text { otherwise }\end{cases}
$$

Note that

$$
\left(\mathcal{A}^{*}\right)_{\sigma} \cup\left(\mathcal{A}^{\prime}\right)_{\sigma}=\left(\mathcal{A}_{\sigma}\right)^{*} \cup\left(\mathcal{A}_{\sigma}\right)^{\prime} \text { and }\left(\mathcal{A}^{*}\right)_{\sigma} \subseteq\left(\mathcal{A}_{\sigma}\right)^{*}
$$

Let $C$ be the set of all $r$-good cyclic orderings of $X$. We have $|C|>0$ because $\tau, \gamma \in C$. As observed in [19], there exists an integer $q>0$ such that any $P \in \mathcal{P}$ meets exactly $q$ $r$-good cyclic orderings of $X$. So $\sum_{\sigma \in C} \Phi(\sigma, P)=q$ for all $P \in \mathcal{P}$. Thus we have 


$$
\begin{aligned}
q\left(\left|\mathcal{A}^{*}\right|+\frac{r}{n^{2}}\left|\mathcal{A}^{\prime}\right|\right) & =\left(\sum_{A^{*} \in \mathcal{A}^{*}} q\right)+\frac{r}{n^{2}}\left(\sum_{A^{\prime} \in \mathcal{A}^{\prime}} q\right) \\
& =\sum_{A^{*} \in \mathcal{A}^{*}} \sum_{\sigma \in C} \Phi\left(\sigma, A^{*}\right)+\frac{r}{n^{2}} \sum_{A^{\prime} \in \mathcal{A}^{\prime}} \sum_{\sigma \in C} \Phi\left(\sigma, A^{\prime}\right) \\
& =\sum_{\sigma \in C}\left(\sum_{A^{*} \in \mathcal{A}^{*}} \Phi\left(\sigma, A^{*}\right)+\frac{r}{n^{2}} \sum_{A^{\prime} \in \mathcal{A}^{\prime}} \Phi\left(\sigma, A^{\prime}\right)\right) \\
& =\sum_{\sigma \in C}\left(\left|\left(\mathcal{A}^{*}\right)_{\sigma}\right|+\frac{r}{n^{2}}\left|\left(\mathcal{A}^{\prime}\right)_{\sigma}\right|\right) \\
& \leq \sum_{\sigma \in C}\left(\left|\left(\mathcal{A}_{\sigma}\right)^{*}\right|+\frac{r}{n^{2}}\left|\left(\mathcal{A}_{\sigma}\right)^{\prime}\right|\right) \quad(\text { by }(1)) \\
& \leq \sum_{\sigma \in C} r \quad(\text { by Lemma 2.1) } \\
& =r|C|
\end{aligned}
$$

and hence $\left|\mathcal{A}^{*}\right|+\frac{r}{n^{2}}\left|\mathcal{A}^{\prime}\right| \leq \frac{r|C|}{q}$. Let $\mathcal{S}$ be the star of $\mathcal{P}$ with centre $(1,1)$. Clearly any cyclic ordering $\sigma$ of $X$ meets exactly $r$ sets containing a fixed element (like $(1,1)$ ), and if $\sigma \in C$ then (by definition of an $r$-good cyclic ordering) these $r$ sets are all in $\mathcal{P}$. This means that $\sum_{S \in \mathcal{S}} \Phi(\sigma, S)=r$ for all $\sigma \in C$. Now as in the above, $q|\mathcal{S}|=\sum_{\sigma \in C} \sum_{S \in \mathcal{S}} \Phi(\sigma, S)$. So $q|\mathcal{S}|=\sum_{\sigma \in C} r=r|C|$, giving $\frac{r|C|}{q}=|\mathcal{S}|$. Therefore $\left|\mathcal{A}^{*}\right|+\frac{r}{n^{2}}\left|\mathcal{A}^{\prime}\right| \leq|\mathcal{S}|$, and hence the inequality in the theorem.

It is straightforward that the bound is attained if one of (i) and (ii) holds. We now prove the converse. If $\mathcal{A}^{\prime}=\emptyset$ then $\left|\mathcal{A}^{*}\right| \leq\left(\begin{array}{c}n-1 \\ r-1\end{array}\right) \frac{(n-1) !}{(n-r) !}$; this is in fact Theorem 1.2, which also tells us that equality holds only if $\mathcal{A}^{*}$ is a star of $\mathcal{P}$. Now suppose $\mathcal{A}^{\prime} \neq \emptyset$ and we have equality in our theorem. So we have equality in (2) and (3). By (1) and the equality in (2), we clearly have

$$
\left(\mathcal{A}^{*}\right)_{\sigma}=\left(\mathcal{A}_{\sigma}\right)^{*} \text { and }\left(\mathcal{A}^{\prime}\right)_{\sigma}=\left(\mathcal{A}_{\sigma}\right)^{\prime} .
$$

The equality in (3) and Lemma 2.1 give us that, for any $\sigma \in C$, if $\left(\mathcal{A}_{\sigma}\right)^{\prime} \neq \emptyset$ then $\left(\mathcal{A}_{\sigma}\right)^{\prime}=\mathcal{P}_{\sigma}$ (and $\left.\left(\mathcal{A}_{\sigma}\right)^{*}=\emptyset\right)$. Thus, by $(4)$,

$$
\text { for any } \sigma \in C \text {, if }\left(\mathcal{A}^{\prime}\right)_{\sigma} \neq \emptyset \text { then }\left(\mathcal{A}^{\prime}\right)_{\sigma}=\mathcal{P}_{\sigma} \text {. }
$$

We now use this together with $\mathcal{A}^{\prime} \neq \emptyset$ to show that $\mathcal{A}^{\prime}=\mathcal{P}$. Let $A_{0} \in \mathcal{A}^{\prime}$. For $i=0,1, \ldots, r$, let $\mathcal{P}^{(i)}:=\left\{P \in \mathcal{P}:\left|P \cap A_{0}\right|=r-i\right\}$; so $\mathcal{P}^{(i)}$ is the family of those sets in $\mathcal{P}$ that have exactly $i$ elements not in $A_{0}$. $\mathcal{P}$ is a disjoint union of the families $\mathcal{P}^{(0)}, \mathcal{P}^{(1)}, \ldots, \mathcal{P}^{(r)}$, and $\mathcal{A}^{\prime}$ contains $\mathcal{P}^{(0)}$ since $\mathcal{P}^{(0)}=\left\{A_{0}\right\}$. Our next step is to show that $\mathcal{A}^{\prime}$ also contains $\mathcal{P}^{(1)}$, and then we go on to show that in fact $\mathcal{A}^{\prime}$ contains each family $\mathcal{P}^{(i)}$.

Let $A_{1}$ be an arbitrary set in $\mathcal{P}^{(1)}$. Let $\left(x_{1}, y_{1}\right), \ldots,\left(x_{r}, y_{r}\right)$ be the distinct elements of $A_{0}$; only one of these is not in $A_{1}$, and we may assume it is $\left(x_{r}, y_{r}\right)$. So $A_{1}=$ $\left\{\left(x_{1}, y_{1}\right), \ldots,\left(x_{r-1}, y_{r-1}\right),(x, y)\right\}$, where $x \in[n] \backslash\left\{x_{1}, \ldots, x_{r-1}\right\}$ and $y \in[n] \backslash\left\{y_{1}, \ldots, y_{r-1}\right\}$. 
Suppose $x \neq x_{r}$ and $y \neq y_{r}$. Let $\pi, \rho \in S_{n}$ such that $\pi(1)=x, \rho(1)=y$, and for $i=1, \ldots, r, \pi(i+1)=x_{i}$ and $\rho(i+1)=y_{i}$. Lemma 2.2 tells us that $\gamma_{\pi, \rho} \in C$. We have $\gamma_{\pi, \rho}(x, y)=\gamma_{\pi, \rho}(\pi(1), \rho(1))=\gamma(1,1)=1, \gamma_{\pi, \rho}\left(x_{1}, y_{1}\right)=\gamma_{\pi, \rho}(\pi(2), \rho(2))=\gamma(2,2)=2, \ldots$, $\gamma_{\pi, \rho}\left(x_{r}, y_{r}\right)=\gamma_{\pi, \rho}(\pi(r+1), \rho(r+1))=\gamma(r+1, r+1)=r+1$. So $\gamma_{\pi, \rho}$ maps the elements of $A_{0}$ to $2, \ldots, r+1$, and $\gamma_{\pi, \rho}$ maps the elements of $A_{1}$ to $1, \ldots, r$; this means that both $A_{0}$ and $A_{1}$ meet $\gamma_{\pi, \rho}$. So $A_{0} \in\left(\mathcal{A}^{\prime}\right)_{\gamma_{\pi, \rho}}$ (recall that $A_{0} \in \mathcal{A}^{\prime}$ ) and $A_{1} \in \mathcal{P}_{\gamma_{\pi, \rho}}$. By (5), we have $\left(\mathcal{A}^{\prime}\right)_{\gamma_{\pi, \rho}}=\mathcal{P}_{\gamma_{\pi, \rho}}$, and hence $A_{1} \in\left(\mathcal{A}^{\prime}\right)_{\gamma_{\pi, \rho}}$. Thus $A_{1} \in \mathcal{A}^{\prime}$.

Now suppose we instead have $x=x_{r}$ or $y=y_{r}$; by symmetry, we may assume $x=x_{r}$. Then $y \neq y_{r}\left(\operatorname{as}(x, y) \notin A_{0}\right)$ and hence $y$ is an element of the set $Y:=$ $[n] \backslash\left\{y_{1}, \ldots, y_{r}\right\}$. Let $y_{r+1}, \ldots, y_{n}$ be the elements of $Y$, taking $y_{r+1}$ to be $y$. So $A_{1}=$ $\left\{\left(x_{1}, y_{1}\right), \ldots,\left(x_{r-1}, y_{r-1}\right),\left(x_{r}, y_{r+1}\right)\right\}$. Let $\pi, \rho, \varrho$ be the members of $S_{n}$ given by $\pi(i)=x_{i}$ for all $i \in[n], \rho(i)=y_{i}$ for all $i \in[n], \varrho(i)=y_{i}$ for all $i \in[n] \backslash\{r, r+1\}, \varrho(r)=y_{r+1}$, $\varrho(r+1)=y_{r}$. Lemma 2.2 tells us that $\gamma_{\pi, \rho}, \gamma_{\pi, \varrho} \in C$. Now let $A^{\prime}$ be the $r$-set in $\mathcal{P}$ consisting of those elements of $[n] \times[n]$ that are numbered $r+2, \ldots, 2 r+1$ by $\gamma_{\pi, \rho}$; so

$$
A^{\prime}= \begin{cases}\left\{\left(x_{r+2}, y_{r+2}\right), \ldots,\left(x_{2 r+1}, y_{2 r+1}\right)\right\} & \text { if } n \geq 2 r+1 ; \\ \left\{\left(x_{r+2}, y_{r+2}\right), \ldots,\left(x_{n}, y_{n}\right),\left(x_{2}, y_{1}\right), \ldots,\left(x_{2 r-n+2}, y_{2 r-n+1}\right)\right\} & \text { if } r+2 \leq n<2 r+1 \\ \left\{\left(x_{2}, y_{1}\right), \ldots,\left(x_{r+1}, y_{r}\right)\right\} & \text { if } n=r+1\end{cases}
$$

Both $A_{0}$ and $A^{\prime}$ meet $\gamma_{\pi, \rho}$, meaning that $A_{0} \in\left(\mathcal{A}^{\prime}\right)_{\gamma_{\pi, \rho}}$ and $A^{\prime} \in \mathcal{P}_{\gamma_{\pi, \rho}}$. By (5), we have $\left(\mathcal{A}^{\prime}\right)_{\gamma_{\pi, \rho}}=\mathcal{P}_{\gamma_{\pi, \rho}}$, and hence $A^{\prime} \in\left(\mathcal{A}^{\prime}\right)_{\gamma_{\pi, \rho}}$. Thus $A^{\prime} \in \mathcal{A}^{\prime}$. Obviously $\gamma_{\pi, \varrho}$ meets $A_{1}$; so $A_{1} \in \mathcal{P}_{\gamma_{\pi, \varrho}}$. Suppose $n \geq r+2$. Then we can immediately see that $\gamma_{\pi, \varrho}$ also meets $A^{\prime}$ (because $2 r-n+1 \leq r-1$ ), meaning that $A^{\prime} \in\left(\mathcal{A}^{\prime}\right)_{\gamma_{\pi, \varrho}}$. As above, it follows by (5) that $A_{1} \in \mathcal{A}^{\prime}$. Now suppose $n=r+1$. Then $\gamma_{\pi, \varrho}\left(x_{r+1}, y_{r}\right)=r+1=n$ and $\gamma_{\pi, \varrho}\left(x_{i+1}, y_{i}\right)=n+i$ for $i=1, \ldots, r-1$. So $\gamma_{\pi, \varrho}$ again meets $A^{\prime}$, and hence we again conclude that $A_{1} \in \mathcal{A}^{\prime}$.

We have therefore shown that $\mathcal{P}^{(1)}$ is contained in $\mathcal{A}^{\prime}$. More importantly, we have in fact shown that

$$
A \in \mathcal{A}^{\prime}, P \in \mathcal{P},|A \cap P|=r-1 \quad \Rightarrow \quad P \in \mathcal{A}^{\prime} .
$$

We claim that, if $i \in\{2, \ldots, r\}$ and $\mathcal{P}^{(i-1)} \subset \mathcal{A}^{\prime}$, then $\mathcal{P}^{(i)} \subset \mathcal{A}^{\prime}$. Since $\mathcal{P}^{(1)} \subset \mathcal{A}^{\prime}$, the claim inductively implies that the families $\mathcal{P}^{(2)}, \ldots, \mathcal{P}^{(r)}$ are also contained in $\mathcal{A}^{\prime}$, and hence $\mathcal{A}^{\prime}=\mathcal{P}$ as required. We conclude the proof by verifying the claim using (6).

Suppose we have $\mathcal{P}^{(i-1)} \subset \mathcal{A}^{\prime}$ for some $i \in\{2, \ldots, r\}$. Let $A_{i}$ be an arbitrary set in $\mathcal{P}^{(i)}$, i.e. $\left|A_{0} \cap A_{i}\right|=r-i$. We may assume $A_{0} \cap A_{i}=A_{0} \backslash\left\{\left(x_{r-i+1}, y_{r-i+1}\right), \ldots,\left(x_{r}, y_{r}\right)\right\}$. For brevity, let $\left(x^{*}, y^{*}\right)$ be $\left(x_{r-i+1}, y_{r-i+1}\right)$. Since $\left(x^{*}, y^{*}\right) \notin A_{i}$, one of the following cases holds: 1. there is no element $(x, y)$ of $A_{i}$ such that $x=x^{*}$ or $y=y^{*}$;

2. there is exactly one element $(x, y)$ of $A_{i}$ such that $x=x^{*}, y \neq y^{*}$ or $x \neq x^{*}, y=y^{*}$;

3. there is exactly one element $(x, y)$ of $A_{i}$ such that $x=x^{*}, y \neq y^{*}$ and exactly one element $\left(x^{\prime}, y^{\prime}\right)$ of $A_{i}$ such that $x^{\prime} \neq x^{*}, y^{\prime}=y^{*}$.

Consider case 1 . Let $(\bar{x}, \bar{y}) \in A_{i} \backslash A_{0}$ and let $A:=\left(A_{i} \backslash\{(\bar{x}, \bar{y})\}\right) \cup\left\{\left(x^{*}, y^{*}\right)\right\}$. Clearly $A \in \mathcal{P}^{(i-1)}$ and hence $A \in \mathcal{A}^{\prime}$. Also, $\left|A \cap A_{i}\right|=r-1$. By $(6), A_{i} \in \mathcal{A}^{\prime}$.

Next, consider case 2. Let $A:=\left(A_{i} \backslash\{(x, y)\}\right) \cup\left\{\left(x^{*}, y^{*}\right)\right\}$. We clearly have $A \in \mathcal{P}^{(i-1)}$, $\left|A \cap A_{i}\right|=r-1$, and hence $A_{i} \in \mathcal{A}^{\prime}$ as in case 1 . 
Finally, consider case 3 . Since $r<n$, we can choose $\bar{y} \in[n]$ such that no element $(a, b)$ of $A_{i}$ has $b=\bar{y}$. Let $A:=\left(A_{i} \backslash\left\{\left(x^{\prime}, y^{\prime}\right)\right\}\right) \cup\left\{\left(x^{\prime}, \bar{y}\right)\right\}$. So $A \in \mathcal{P}$ and $\left|A \cap A_{i}\right|=r-1$. If $\left(x^{\prime}, \bar{y}\right) \in$ $A_{0}$ then $A \in \mathcal{P}^{(i-1)}$ (as $\left.\left(x^{\prime}, \bar{y}\right) \notin A_{0} \cap A_{i}\right)$ and hence $A_{i} \in \mathcal{A}^{\prime}$. So suppose $\left(x^{\prime}, \bar{y}\right) \notin A_{0}$. Then, by taking $A^{\prime}:=(A \backslash\{(x, y)\}) \cup\left\{\left(x^{*}, y^{*}\right)\right\}=\left(A_{i} \backslash\left\{(x, y),\left(x^{\prime}, y^{\prime}\right)\right\}\right) \cup\left\{\left(x^{*}, y^{*}\right),\left(x^{\prime}, \bar{y}\right)\right\}$, we clearly get that $A^{\prime} \in \mathcal{P}^{(i-1)},\left|A^{\prime} \cap A\right|=r-1$, and hence $A \in \mathcal{A}^{\prime}$, which in turn implies $A_{i} \in \mathcal{A}^{\prime}$ since $\left|A \cap A_{i}\right|=r-1$.

Therefore, $\mathcal{P}^{(i)}$ is contained in $\mathcal{A}^{\prime}$ as we claimed.

Lemma 2.3 Let $r<n$. Suppose $\emptyset \neq \mathcal{F} \subseteq \mathcal{P}_{n, r}$ such that, for any $A \in \mathcal{F}$ and $B \in \mathcal{P}_{n, r}$ with $A \cap B=\emptyset, B \in \mathcal{F}$. Then $\mathcal{F}=\mathcal{P}_{n, r}$.

Proof. The result is trivial for $n \leq 2$, so we assume $n \geq 3$. For any positive integer $z$, let $\theta_{z}$ : $\mathcal{P}_{n, r} \rightarrow \mathcal{P}_{n, r}$ be the translation operation defined by $\theta_{z}(A):=\left\{\left(x, y+z \bmod ^{*} n\right):(x, y) \in A\right\}$ (that is, $\theta_{z}$ shifts the points in $A$ upwards by $z$ positions in the cyclic sense). Let $F_{1}$ be a set in $\mathcal{F}$. For each $z \in[n-1]$, let $F_{z+1}:=\theta_{z}\left(F_{1}\right)$. The sets $F_{1}, F_{2}, \ldots, F_{n}$ are disjoint, and hence the conditions of the lemma tell us that they are all in $\mathcal{F}$. Since a set $P$ in $\mathcal{P}_{n, r}$ can intersect at most $r$ disjoint sets (simply because it has $r$ elements), there exists $j \in[n]$ such that $P \cap F_{j}=\emptyset$, meaning that $P \in \mathcal{F}$. Hence the result.

Proof of Theorem 1.7. The result for $r=n$ is Theorem 1.5, so we consider $r<n$. It is easy to check that the bound in the theorem is attained in each of the cases (i), (ii), (iii). We now show that $\sum_{i=1}^{k}\left|\mathcal{A}_{i}\right|$ is a maximum only in those cases.

Let $\mathcal{A}=\bigcup_{i=1}^{k} \mathcal{A}_{i}$. Clearly $\mathcal{A}^{*}=\bigcup_{i=1}^{k} \mathcal{A}_{i}^{*}$ and $\mathcal{A}^{\prime}=\bigcup_{i=1}^{k} \mathcal{A}_{i}^{\prime}$. Suppose that for some distinct $i$ and $j$ in $[k], \mathcal{A}_{i}^{\prime}$ and $\mathcal{A}_{j}^{\prime}$ have a common member $A$. Having $A \in \mathcal{A}_{i}^{\prime}$ means that there exists a set $A_{i}$ in $\mathcal{A}_{i}^{\prime}$ that does not intersect $A$, but this contradicts the crossintersection condition because $A \in \mathcal{A}_{j}$. So the families $\mathcal{A}_{1}^{\prime}, \ldots, \mathcal{A}_{k}^{\prime}$ are pair-wise disjoint, and hence $\left|\mathcal{A}^{\prime}\right|=\sum_{i=1}^{k}\left|\mathcal{A}_{i}^{\prime}\right|$. Applying Theorem 1.6, we therefore get

$$
\begin{aligned}
\sum_{i=1}^{k}\left|\mathcal{A}_{i}\right| & =\sum_{i=1}^{k}\left|\mathcal{A}_{i}^{\prime}\right|+\sum_{i=1}^{k}\left|\mathcal{A}_{i}^{*}\right| \leq\left|\mathcal{A}^{\prime}\right|+k\left|\mathcal{A}^{*}\right|=\frac{n^{2}}{r}\left(\frac{r}{n^{2}}\left|\mathcal{A}^{\prime}\right|+\left|\mathcal{A}^{*}\right|+\frac{k r-n^{2}}{n^{2}}\left|\mathcal{A}^{*}\right|\right) \\
& \leq \frac{n^{2}}{r}\left(\left(\begin{array}{c}
n-1 \\
r-1
\end{array}\right) \frac{(n-1) !}{(n-r) !}+\frac{k r-n^{2}}{n^{2}}\left|\mathcal{A}^{*}\right|\right)=\left(\begin{array}{c}
n \\
r
\end{array}\right) \frac{n !}{(n-r) !}+\left(k-\frac{n^{2}}{r}\right)\left|\mathcal{A}^{*}\right| .
\end{aligned}
$$

Suppose $k<\frac{n^{2}}{r}$. Then $\sum_{i=1}^{k}\left|\mathcal{A}_{i}\right| \leq\left(\begin{array}{l}n \\ r\end{array}\right) \frac{n !}{(n-r) !}$, and equality holds if and only if $\mathcal{A}^{*}=\emptyset$ and $\mathcal{A}=\mathcal{A}^{\prime}=\mathcal{P}_{n, r}$. Now suppose $\mathcal{A}=\mathcal{P}_{n, r}$, and let $i \in[k]$ such that $\mathcal{A}_{i} \neq \emptyset$. Together with the cross-intersection condition, this implies that, if $A \in \mathcal{A}_{i}$ and $B$ is a set in $\mathcal{P}_{n, r}$ that does not intersect $A$, then $B$ has to be in $\mathcal{A}_{i}$. So the conditions of Lemma 2.3 hold for $\mathcal{A}_{i}$, and hence $\mathcal{A}_{i}=\mathcal{P}_{n, r}$. Due to the cross-intersection condition, this forces any other family $\mathcal{A}_{j}$ to be empty. Hence (i).

Next, suppose $k>\frac{n^{2}}{r}$. Then, by (7) and Theorem 1.6,

$$
\sum_{i=1}^{k}\left|\mathcal{A}_{i}\right| \leq\left(\begin{array}{l}
n \\
r
\end{array}\right) \frac{n !}{(n-r) !}+\left(k-\frac{n^{2}}{r}\right)\left(\begin{array}{c}
n-1 \\
r-1
\end{array}\right) \frac{(n-1) !}{(n-r) !}=k\left(\begin{array}{c}
n-1 \\
r-1
\end{array}\right) \frac{(n-1) !}{(n-r) !}
$$


and equality holds if and only if $\mathcal{A}_{1}^{*}=\ldots=\mathcal{A}_{k}^{*}=\mathcal{A}^{*}$ and $\left|\mathcal{A}^{*}\right|=\left(\begin{array}{c}n-1 \\ r-1\end{array}\right) \frac{(n-1) !}{(n-r) !}=|\mathcal{A}|$. Now Theorem 1.1 tells us that $\left|\mathcal{A}^{*}\right|=\left(\begin{array}{c}n-1 \\ r-1\end{array}\right) \frac{(n-1) !}{(n-r) !}$ if and only if $\mathcal{A}^{*}$ is a star of $\mathcal{P}_{n, r}$. Hence (ii).

Finally, suppose $k=\frac{n^{2}}{r}$. Then, by (7),

$$
\frac{r}{n^{2}} \sum_{i=1}^{k}\left|\mathcal{A}_{i}\right| \leq\left|\mathcal{A}^{*}\right|+\frac{r}{n^{2}}\left|\mathcal{A}^{\prime}\right| \leq\left(\begin{array}{c}
n-1 \\
r-1
\end{array}\right) \frac{(n-1) !}{(n-r) !}
$$

If $\mathcal{A}^{*}=\emptyset$ then $\mathcal{A}$ is as in the case $k<\frac{n^{2}}{r}$, and if $\mathcal{A}^{*} \neq \emptyset$ then it is immediate from Theorem 1.6 that $\mathcal{A}^{*}$ is as in the case $k>\frac{n^{2}}{r}$. Hence the result.

\section{References}

[1] B. Bollobás and I. Leader, An Erdôs-Ko-Rado theorem for signed sets, Comput. Math. Appl., 34(1997), pp. 9-13.

[2] P. Borg, A short proof of a cross-intersection theorem of Hilton, Discrete Math. 309 (2009), 4750-4753.

[3] P. Borg, Cross-intersecting families of permutations, J. Combin. Theory Ser. A, in press; doi:10.1016/j.jcta.2009.07.003.

[4] P. Borg, Intersecting and cross-intersecting families of labeled sets, Electron. J. Combin., 15(2008), \#N9.

[5] P. Borg and I. Leader, Multiple cross-intersecting families of signed sets, J. Combin. Theory Ser. A, to appear.

[6] P.J. Cameron and C.Y. Ku, Intersecting families of permutations, European J. Combin., 24(2003), pp. 881-890.

[7] D.E. Daykin, Erdôs-Ko-Rado from Kruskal-Katona, J. Combin. Theory Ser. A, 17(1974), pp. 254-255.

[8] M. Deza and P. Frankl, On the maximum number of permutations with given maximal or minimal distance, J. Combin. Theory Ser. A, 22(1977), pp. 352-360.

[9] M. Deza and P. Frankl, The Erdős-Ko-Rado theorem - 22 years later, SIAM J. Algebraic Discrete Methods, 4(1983), pp. 419-431.

[10] D. Ellis, A proof of the Cameron-Ku conjecture, arXiv:0807.3118v2.

[11] D. Ellis, E. Friedgut, H. Pilpel, Intersecting families of permutations, submitted.

[12] P. Erdős, C. Ko and R. Rado, Intersection theorems for systems of finite sets, Quart. J. Math. Oxford (2), 12(1961), pp. 313-320. 
[13] P. Frankl, The shifting technique in extremal set theory, in: C. Whitehead (Ed.), Combinatorial Surveys, Cambridge Univ. Press, London/New York, 1987, pp. 81-110.

[14] A.J.W. Hilton, An intersection theorem for a collection of families of subsets of a finite set, J. London Math. Soc. (2), 15(1977), pp. 369-376.

[15] A.J.W. Hilton and E.C. Milner, Some intersection theorems for systems of finite sets, Quart. J. Math. Oxford (2), 18(1967), pp. 369-384.

[16] G.O.H. Katona, A simple proof of the Erdős-Chao Ko-Rado theorem, J. Combin. Theory Ser. B, 13(1972), pp. 183-184.

[17] G.O.H. Katona, A theorem of finite sets, in: Theory of Graphs, Proc. Colloq. Tihany, Akadémiai Kiadó, 1968, pp. 187-207.

[18] J.B. Kruskal, The number of simplices in a complex, in: Mathematical Optimization Techniques, University of California Press, Berkeley, California, 1963, pp. 251-278.

[19] C.Y. Ku and I. Leader, An Erdôs-Ko-Rado theorem for partial permutations, Discrete Math., 306(2006), pp. 74-86.

[20] B. Larose and C. Malvenuto, Stable sets of maximal size in Kneser-type graphs, European J. Combin., 25(2004), pp. 657-673.

[21] Y.-S. Li and J. Wang, Erdôs-Ko-Rado-type theorems for colored sets, Electron. J. Combin., 14(2007), \#R1. 